\title{
Measurements and Classifications in Spine Imaging
}

\author{
Simone Waldt, $\mathrm{MD}^{1} \quad$ Alexandra Gersing, $\mathrm{MD}^{2} \quad$ Melanie Brügel, $\mathrm{MD}^{1}$ \\ ${ }^{1}$ Department of Radiology, Technische Universität München, Munich, \\ Germany \\ ${ }^{2}$ Department of Neuroradiology, Technische Universität München, \\ Munich, Germany \\ Address for correspondence Simone Waldt, MD, Department of \\ Radiology, Technische Universität München, Ismaninger Str. 22, 81675 \\ Munich, Germany (e-mail: simone.waldt@tum.de). \\ Semin Musculoskelet Radiol 2014;18:219-227.
}

\begin{abstract}
Imaging criteria and radiologic measurements play a key role in the diagnosis of spinal diseases. In addition, they often create the basis of classification systems that determine the severity of the disease and thereby enable a stage-related therapy. A clearly defined nomenclature for imaging findings as well as standardized and thoroughly evaluated

Keywords

- spine

- measurement

- MRI

- scoliosis

- degenerative changes methods of measurement are necessary to achieve a sufficiently high diagnostic accuracy. Various specialized committees dealing with the diagnosis of spinal diseases have made efforts within the last years to develop diagnostic standards. This review provides an overview of radiologic measurements and classification systems that are currently used for the diagnosis of scoliosis and degenerative diseases of the lumbar spine.
\end{abstract}

\section{Scoliosis}

To select the therapeutic regime for patients with idiopathic scoliosis, it is necessary to analyze important imaging-based factors concerning the severity and the prognosis of the disease. The extent of the curve (Cobb angle, secondary Ferguson angle), the rotation of the apical vertebra (NashMoe method of vertebral rotation), the topographic classification, and the remaining growth potential (Risser sign) are relevant factors that are also part of the basic examination requirements of the Scoliosis Research Society (SRS). ${ }^{1,2}$ Despite some limitations, the classification of the entire malposition (Lenke classification) is the most established classification, which is currently used as the foundation for determining the fusing levels for surgical treatment of idiopathic scoliosis. $^{3}$

\section{Cobb Angle}

The standing anteroposterior (AP) or posteroanterior (PA) radiograph of the full spine is the basic tool for the radiologic quantification of coronal spine deformity. To achieve a high degree of standardization, the SRS has defined the Cobb measurement as the reference method. ${ }^{1}$ The Cobb angle is measured between the two end vertebrae of a curve, which are defined as the most tilted vertebrae at the top and at the bottom of a curve. The Cobb angle is formed by the intersection of the following lines: a line perpendicular to the superior end plate of the cephalad end vertebra and a line perpendicular to the inferior end plate of the caudal end $\operatorname{vertebra}^{4}$ (-Fig. 1).

\section{Ferguson Angle}

Alternatively, the degree of the coronal spine deformity can be determined by using the Ferguson angle. ${ }^{5}$ In accordance with the measurement of the Cobb angle, the two end vertebrae of a curve are identified. In addition, the apical vertebra of the curve is determined. Traditionally the apical vertebra was defined as the most rotated and deformed but least tilted vertebra. The current definition of the SRS is simplified and describes the apical vertebra as the vertebra most deviated laterally from the central sacral vertical line (CSVL), a vertical line that passes through the center of the first sacral segment. ${ }^{1}$ The measuring points identified are the midpoints of the end vertebrae bodies and the apical vertebra body, as shown in $\boldsymbol{\sim}$ Fig. 2 . The angle formed between the lines
Issue Theme Spine; Guest Editor, Mara Epermane, MD
Copyright $\odot 2014$ by Thieme Medical Publishers, Inc., 333 Seventh Avenue, New York, NY 10001, USA. Tel: +1(212) 584-4662.
DOI http://dx.doi.org/ 10.1055/s-0034-1375565. ISSN 1089-7860. 


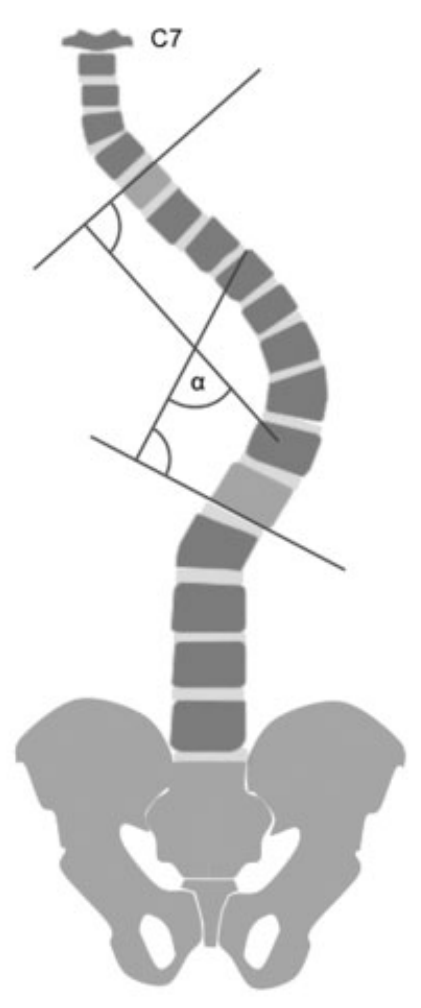

Fig. 1 Measurement of the Cobb angle ( $\alpha$ ) on a full-spine radiograph. The Cobb angle $(\alpha)$ is measured between the two vertebrae that are inclined most toward the concavity of the curve.

connecting the midpoints of the end vertebrae and the apical vertebra equates to the Ferguson angle ${ }^{5}$ (-Fig. 2).

\section{Risser Sign}

The degree of ossification present in the iliac apophysis is semiquantitatively assessed with the Risser sign, which allows an estimation of the skeletal maturity in patients. ${ }^{6}$ Because the ossification of the iliac apophyses occurs approximately parallel to the ossification of the vertebral ring apophyses, the remaining growth potential of the spinal column can be assessed by using the Risser sign. ${ }^{6}$ Idiopathic scoliosis tends to progress during the adolescent growth spurt. Prognosis is therefore more favorable in patients with advanced skeletal maturity and consequently with higher Risser stages. Because there was no staging performed in the original publication by Risser, differently defined staging models can be found in the literature. The classification system presented in - Fig. $\mathbf{3}$ is widely used and has been recommended by the SRS. ${ }^{1}$ The ossification of the iliac apophysis is radiologically detectable at the age of 12 to 15 years. It begins anterolaterally and progresses posteromedially. The apophysis starts to fuse with the os ilium roughly after the adolescent growth spurt and is generally completed after $\sim 3$ years (21-25 years of age). Besides the Risser sign, other radiologic methods exist to assess skeletal maturity. However, because the Risser sign is determined on

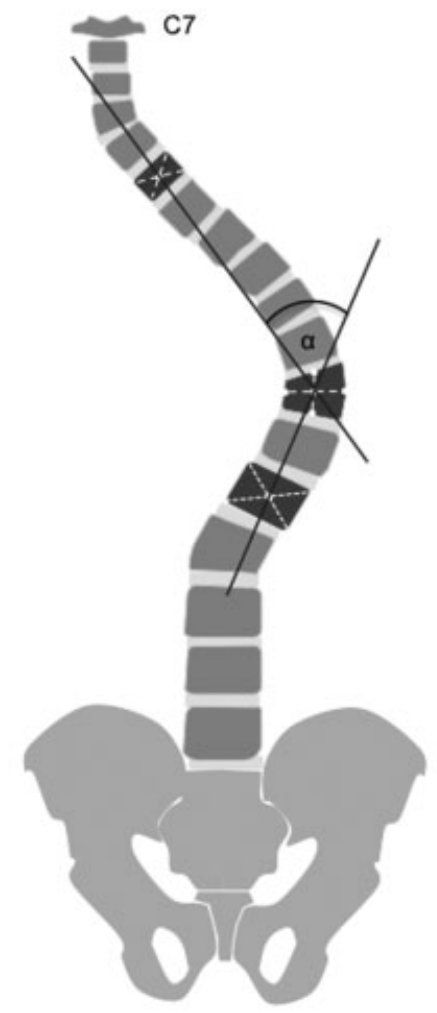

Fig. 2 Measurement of the Ferguson angle on a full-spine radiograph. Lines between the centers of the end vertebrae and the apical vertebra intersect at the Ferguson angle.

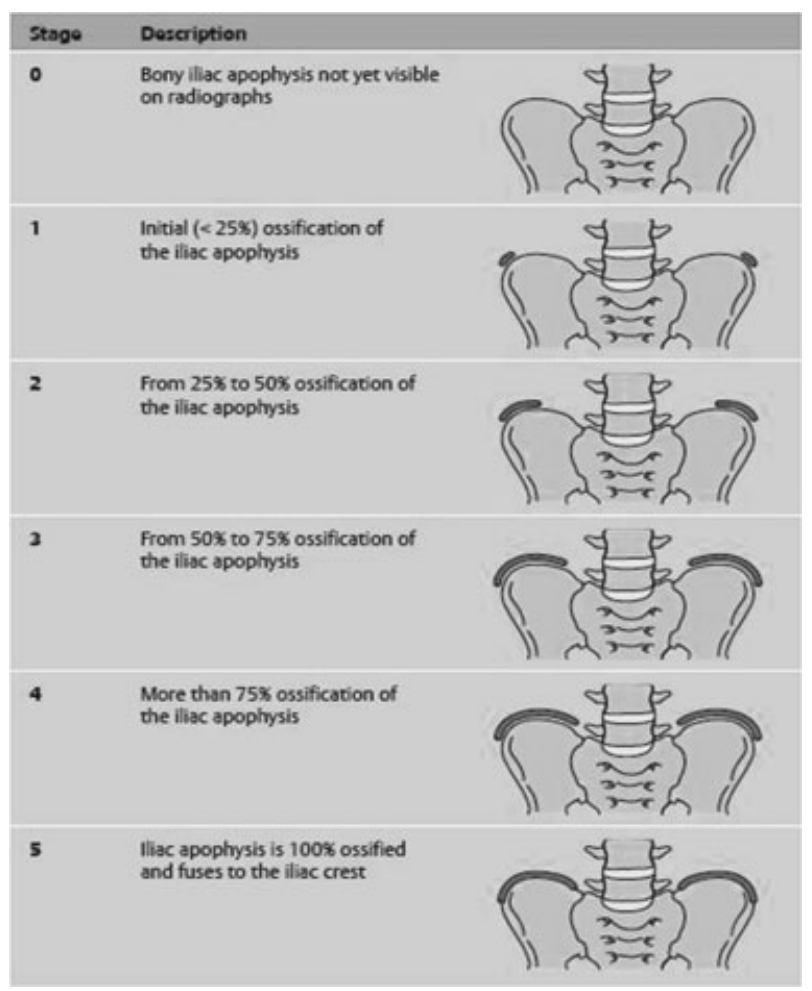

Fig. 3 Assessment of skeletal maturity with the Risser sign. 


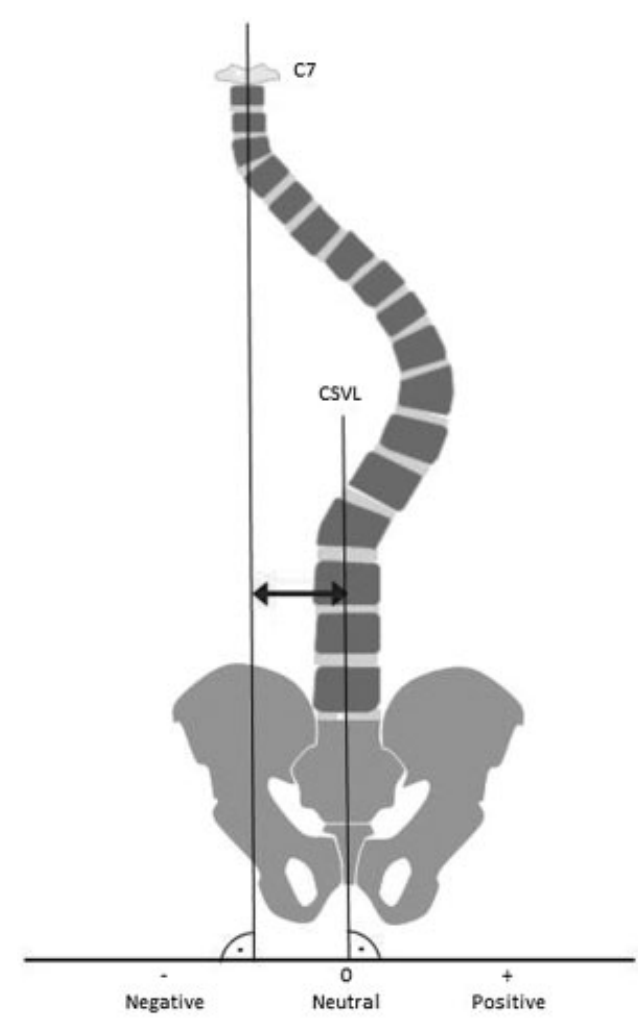

Fig. 4 Determination of the coronal trunk balance, defined as the distance between a vertical plumb line dropped downward from $C 7$ and the central sacral vertical line (CSVL).

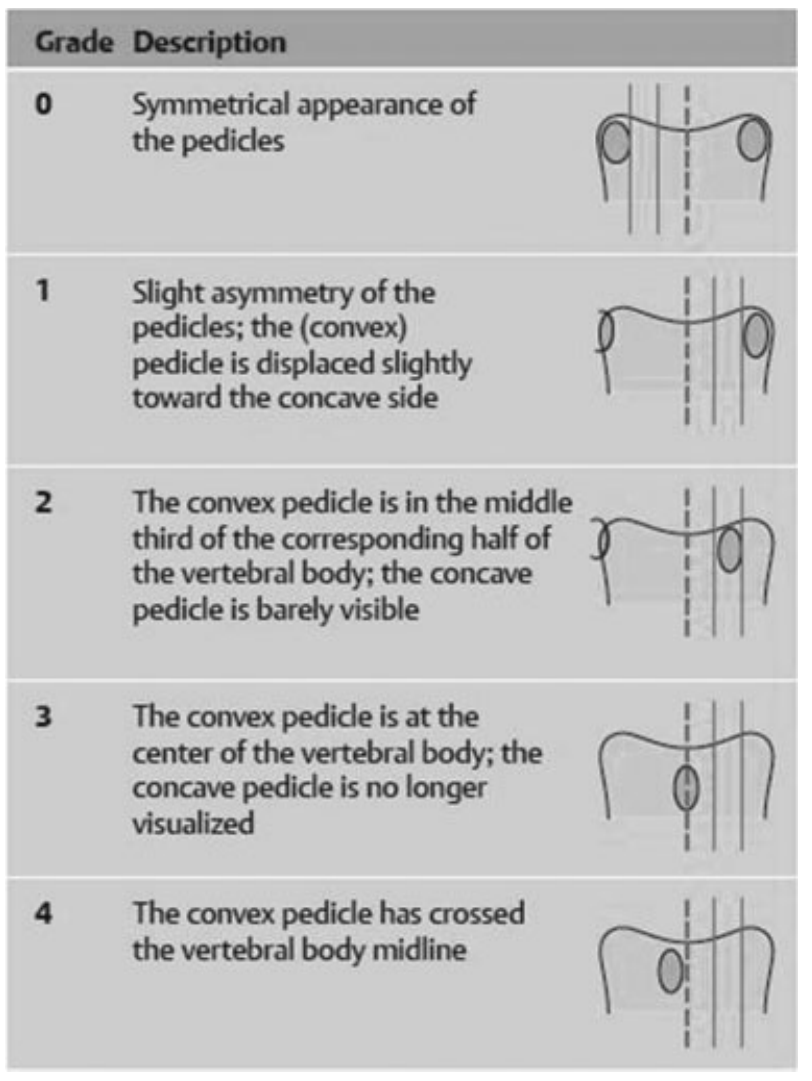

Fig. 5 Nash-Moe method of vertebral rotation. Displacement of the pedicles with respect to segments of the vertebral body is used to grade vertebral rotation. the full spine radiograph, the advantage is that no additional radiation exposure is required.

\section{Coronal Trunk Balance}

The lateral deviation of the trunk in the frontal plane is an indicator for the overall balance of the spinal column. ${ }^{7}$ On a full-spine radiograph, a vertical plumb line is dropped downward from the center of the $\mathrm{C} 7$ vertebral body to the true horizontal. The distance between this line and the CSVL, which is a vertical line drawn through the center of the first sacral segment, equals the coronal trunk balance (-Fig. 4). ${ }^{8} \mathrm{~A}$ negative value is measured when the vertical plumb line deviates to the left; a positive value is measured when the vertical plumb line deviates to the right. ${ }^{8}$

\section{Nash-Moe Method of Vertebral Rotation}

The degree of rotation of the apical vertebra can be estimated by the method of Nash and Moe. ${ }^{9}$ The apical vertebra of a curve is the vertebra most rotated and laterally displaced. Rotation causes displacement of both pedicles of the apical vertebra toward the concave side of a curve. According to Nash and Moe, the vertebral body is divided into six segments and the rotation of the pedicle is assessed on a 5-point scale as shown in - Fig. . $^{9}$

\section{Topographic Classification}

The simplest classification of scoliosis is based on the level of the apical vertebra. The following classification was developed in accordance with the SRS with the apical vertebra defined as the most laterally displaced vertebra of a curve (-Table 1). ${ }^{1}$

\section{Lenke Classification}

One of the main goals pursued with the implementation of the Lenke classification was to create a classification that allows the assessment of scolioses with a high inter- and intraobserver reliability. ${ }^{10}$ Since the method was introduced in 2001, this classification has been established as the basis for many orthopedists planning surgical procedures. ${ }^{3}$ The basis of the Lenke classification comprises coronal and lateral full-spine radiographs as well as the right and left supine sidebending views. ${ }^{10}$ Three essential factors are taken into consideration in the Lenke classification: curve type (1 through 6), lumbar spine modifier (A, B, or C) and the sagittal thoracic modifier $(-, \mathrm{N}$, or + ) (-Fig. 6 ).

To determine the curve type, the Cobb angle for each curve is measured on the coronal full-spine radiograph. The greatest curve is defined as the major curve. The topographic classification of the curves is in accordance with the requirements of the SRS ( - Table $\mathbf{1}$ ). The additional minor curves are evaluated by their flexibility and are categorized into structural and nonstructural curves. A structural minor curve is present if a curve $>25$ degrees remains in the side-bending views or the kyphosis angle remains $>20$ degrees in the lateral projection. ${ }^{10}$ Six curve types can be distinguished with these parameters.

The degree of the lumbar deformity, which has a great impact on the overall balance of the vertebral column and 


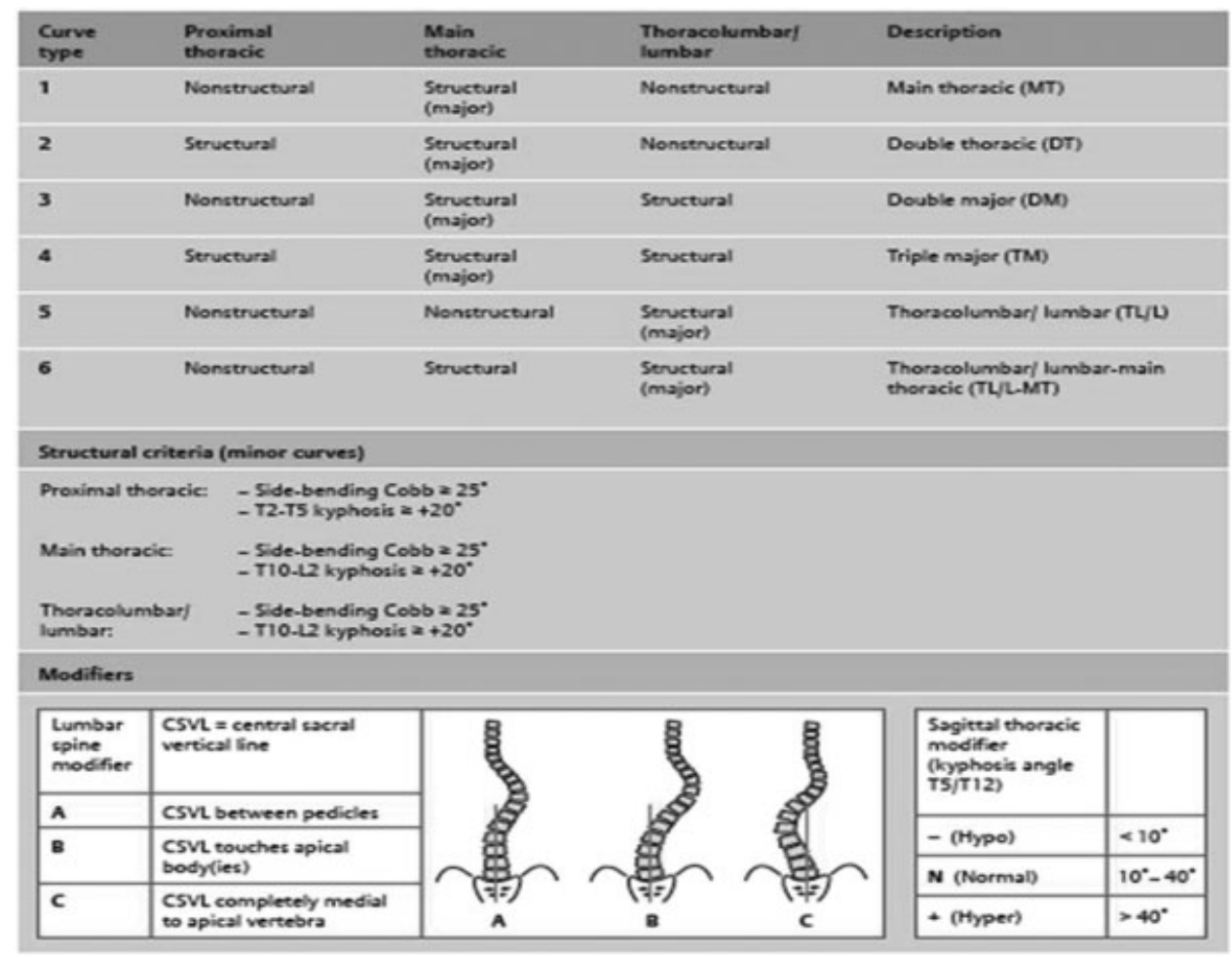

Fig. 6 Synopsis of the Lenke classification.

affects the proximal curve, is evaluated with the lumbar spine modifier. ${ }^{10}$ For this purpose the CSVL is drawn as a vertical line to the true horizontal through the center of the upper sacral segment margin on an upright coronal radiograph. ${ }^{10}$ The vertebral body (lower thoracic or lumbar spine) that is divided equally by this line is defined as a stable vertebral body and the vertebra that is most laterally displaced from this line is defined as an apical vertebral body. Considering the course of the CSVL with regard to the stable and apical vertebrae, three subtypes of the lumbar spine modifier are defined (-Fig. 6 ).

The sagittal thoracic modifier is determined by measuring the thoracic kyphotic angle on the standing lateral radiograph. This means the angle between the superior end plate of T5 and the lower end plate of T12 is measured. In patients with adolescent idiopathic scoliosis, the kyphotic angles are often diminished (mean value $\sim 30$ degrees; normal range: 10-40 degrees). ${ }^{10,11}$ A value $<10$ degrees is termed as hypokyphosis with a minus symbol. Normal values, abbreviated as $\mathrm{N}$, range between 10 and 40 degrees, and values $>40$

Table 1 Topographic classification of scoliosis according to the Scoliosis Research Society

\begin{tabular}{|l|l|}
\hline Level of apical vertebra & Type of scoliosis \\
\hline T2-T11/12 & Thoracic scoliosis \\
\hline T12-L1 & Thoracolumbar scoliosis \\
\hline L1-L2 disk-L4 & Lumbar scoliosis \\
\hline L5 or below & Lumbosacral \\
\hline
\end{tabular}

degrees are defined as hyperkyphosis and marked with an adjacent plus symbol. After evaluating all three parameters, the Lenke type is encoded with a three-digit permutation $\left(\right.$ e.g., $1 \mathrm{~B}+$ ) $\left(\right.$-Fig. 6). ${ }^{10}$

\section{Degenerative Lumbar Spine Disease}

\section{Intervertebral Disk Heights}

The knowledge of the physiologic sequence of the heights of the intervertebral disks on radiographs of the lumbar spine is a valuable tool to diagnose segmental pathologies with conventional radiographs. Dihlmann und Bandick postulated that the physiologic height of the intervertebral disk increases slightly from L1-L2 to L4-L5, but segment L5-S1 decreases or stays unchanged (-Fig. 7). ${ }^{12}$ Later studies describe that in a fair amount of cases a slight continuous increase of the segment L5-S1 is normal. ${ }^{13}$ The following order can be used as a rule of thumb for the physiologic intervertebral disk height sequence:

$$
\text { L1-L2 }<\text { L2-L3 }<\text { L3-L4 }<\text { L4-L5 } \geq \text { or }<\text { L5-S1 }{ }^{13}
$$

\section{Lumbar Stenosis}

Radiologic diagnosis of the lumbar spinal stenosis is complicated by a large number of imprecisely defined and inadequately evaluated methods of measurement. Although the interdisciplinary application of few validated methods of measurement would be favorable to achieve a high level of standardization, no unified guideline or consensus has been established so far. ${ }^{14-16}$ Instead, a survey among 


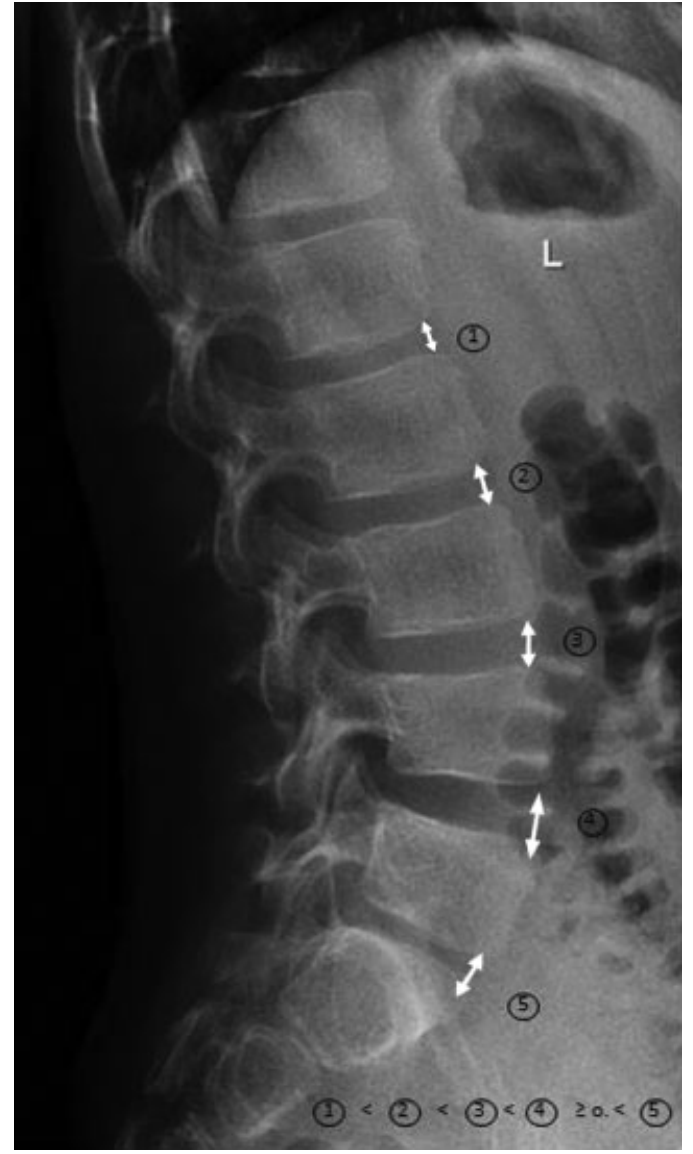

Fig. 7 Physiologic sequence of intervertebral disk heights. $\mathrm{L} 1-\mathrm{L} 2<\mathrm{L} 2-\mathrm{L} 3<\mathrm{L} 3-\mathrm{L} 4<\mathrm{L} 4-\mathrm{L} 5 \geq$ or $<\mathrm{L} 5-\mathrm{S} 1$.

musculoskeletal and neuroradiologic experts showed that no broadly accepted quantitative criteria for the diagnosis of spinal stenosis exist. Actually these experts attributed greater importance to qualitative compared with quantitative criteria. ${ }^{14}$ Only the AP diameter of the spinal canal on the transverse images ( $\mathbf{- F i g . ~ 8 ) ~ w a s ~ c o n s i d e r e d ~ a n ~ e s t a b l i s h e d ~}$ and important criterion with a high level of agreement. However, the five quantitative parameters that were the most highly esteemed for the diagnosis of spinal canal stenosis with magnetic resonance imaging (MRI) in this survey are listed in the following. ${ }^{14}$ Nonexperienced radiologists in particular benefit from having access to quantitative measurement tools for the diagnostic evaluation of spinal stenosis.

\section{Anteroposterior Diameter of Osseous Spinal Canal}

The AP diameter of the osseous spinal canal represents the midsagittal diameter and is measured on transverse or sagittal images between the posterior margin of the vertebral body and the anterior border of the posterior vertebral elements (-Fig. 8). A cutoff value of $11 \mathrm{~mm}$ is considered the threshold for spinal canal stenosis at the level of L3 and L4. ${ }^{14}$

\section{Midsagittal Diameter of Dural Sac}

Next to the measurement of the absolute diameter of the dural sac (threshold value for spinal canal stenosis: $12 \mathrm{~mm}$ ),

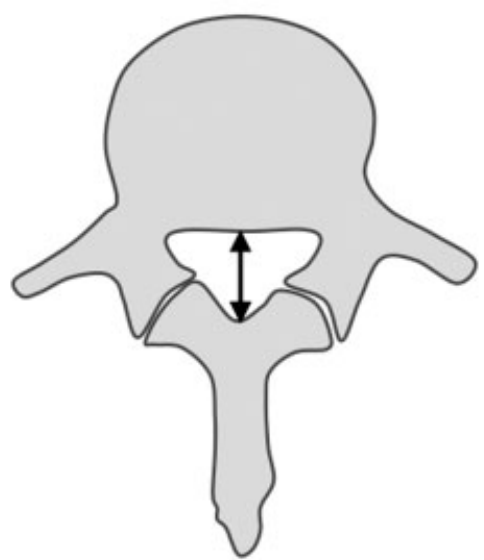

Fig. 8 The anteroposterior diameter of the spinal canal is measured in the midline between the posterior margin of the vertebral body and the anterior border of the vertebral arch.

the relative width can be determined alternatively. ${ }^{14}$ Herzog et al determine the degree of the dural sac compression by comparing the AP diameter of the dural sac on the level of the stenosis with the normal width of the dural sac. ${ }^{17}$ The measurement is performed on midsagittal MR images. This means the AP diameter of the dural sac on the level of the stenosis (disk level) as well as the AP diameter of the dural sac on the midvertebral levels (pedicular level) of the neighboring vertebral bodies are measured (-Fig. 9). The arithmetic average of both midvertebral diameters is considered the normal dural sac width. The percentage of dural sac compression is then calculated by dividing the AP diameter on the level of the stenosis by the normal width of the dural sac. ${ }^{17}$ Implementing this parameter, Herzog and colleagues graded the compression of the dural sac (-Table 2). ${ }^{17}$

\section{Cross-Sectional Area of Dural Sac}

The surface area of the dural sac can be fairly easily measured on transverse images on a computer-based workstation, so that the diagnosis of a spinal canal stenosis can be made with a defined area reduction. Different methods of measurement and thresholds concerning this issue have been described in the literature. ${ }^{14,18,19}$ Transverse images angled vertically to the dural sac are required for this measurement (-Fig. 10). Moreover, T2-weighted images, which provide a good contrast between the dural sac and the neighboring canal structures, are recommended. The threshold of $100 \mathrm{~mm}^{2}$ is an established and expressive value for the diagnosis of a spinal canal stenosis. In the study of Hamanishi et al, a surface area reduction $<100 \mathrm{~mm}^{2}$ on two intervertebral levels was strongly associated with the clinical picture of a claudicatio spinalis. $^{18}$

\section{Lateral Recess Height}

The reduction of the lateral recess height of $<3 \mathrm{~mm}$ is considered evidence of a lateral recess stenosis. ${ }^{14,20,21}$ The measurement is conducted on transverse images (optionally computed tomography [CT] or MRI). ${ }^{14}$ The distance between the most anterior point of the superior articular facet and the 
a
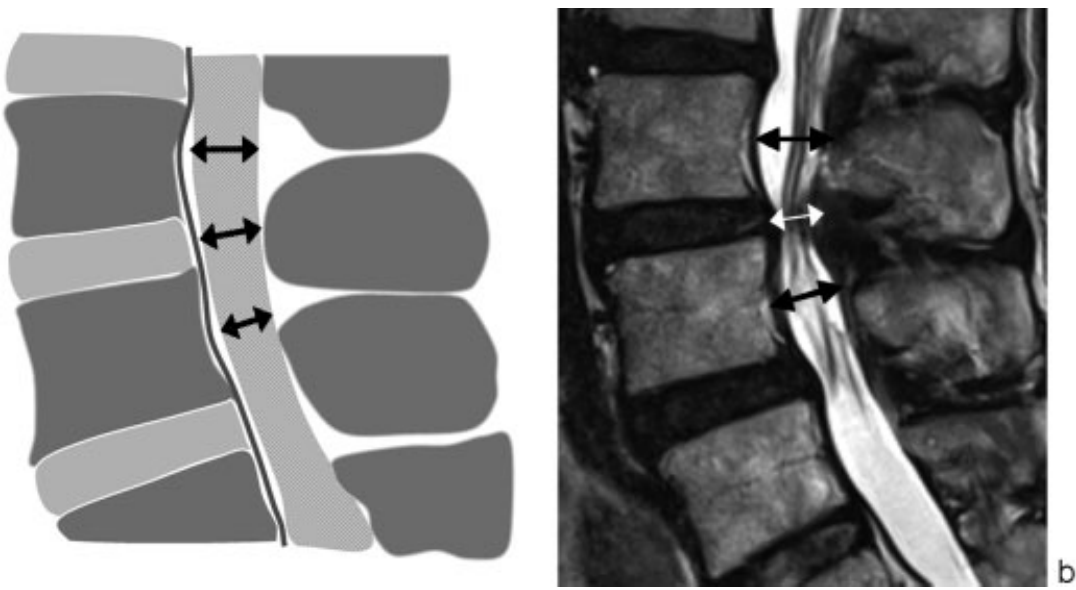

Fig. 9 Measurement of dural sac compression by the method of Herzog et al. (a) Schematic drawing; (b) sagittal T2-weighted TSE image.

a
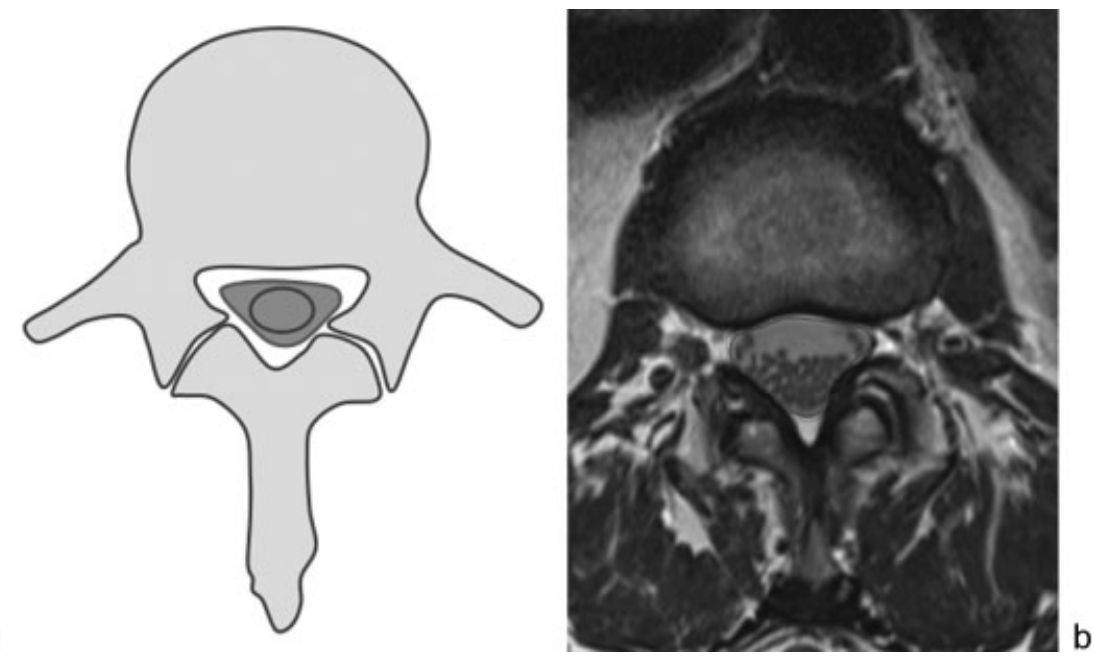

Fig. 10 Assessment of the surface area of the dural sac on transverse images that are oriented perpendicularly to the longitudinal axis of the dural sac. (a) Schematic drawing; (b) transverse T2-weighted TSE image.

posterior margin of the vertebral body is measured (-Fig. 11).

\section{Diameter of Foramen}

The diameter of the neural foramina can be assessed best on sagittal MR or also CT images (-Fig. 12). The maximal anteroposterior diameter of the foramen is measured on sagittal images through the foraminal zone (see later). Alternatively, the measurements can be performed on transverse images. A measurement below the threshold of $3 \mathrm{~mm}$ indicates the presence of a foraminal stenosis. The width of the neural foramen has only been evaluated in a small number of studies. ${ }^{14,15,22}$

\section{Nomenclature and Classification of Lumbar Disk Pathology}

A panel of experts with representatives of the North American Spine Society, the American Society of Spine Radiology, and the American Society of Neuroradiology developed a uniform nomenclature and classification of the pathologies of the lumbar intervertebral disks. ${ }^{23}$ The previous widely used pathoanatomical classification of dorsal intervertebral disk protrusions was deliberately omitted because the anatomical elements (nucleus pulposus, posterior longitudinal ligament, etc.) that are relevant for the categorization of disk displacement cannot always be reliably identified on MR and CT images.

Table 2 Grading dural sac compression by the Herzog method

\begin{tabular}{|l|l|l|l|}
\hline & Grade 1 & Grade 2 & Grade 3 \\
\hline Anterior thecal sac compression & $<15 \%$ & $15-30 \%$ & $>30 \%$ \\
\hline Posterior thecal sac compression & $<10 \%$ & $10-20 \%$ & $>20 \%$ \\
\hline
\end{tabular}



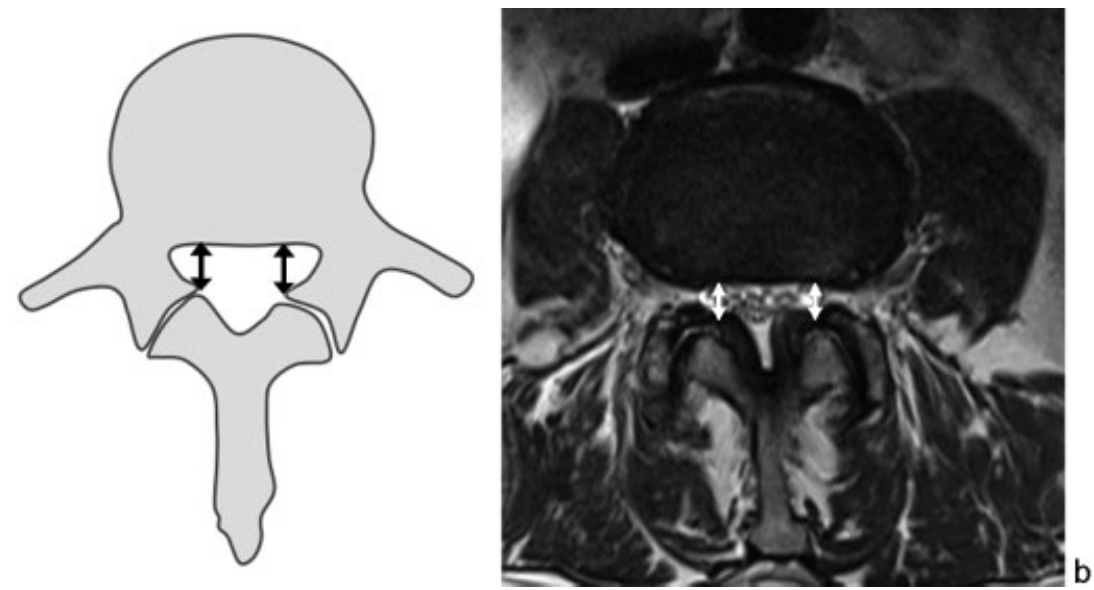

Fig. 11 Assessment of lateral recess height between the most anterior point of the superior articular facet and the posterior margin of the vertebral body. (a) Schematic drawing; (b) transverse T2-weighted TSE image.

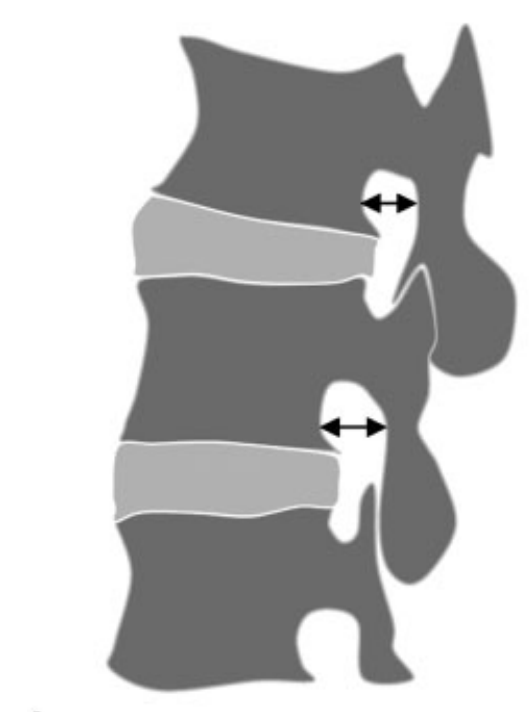

a

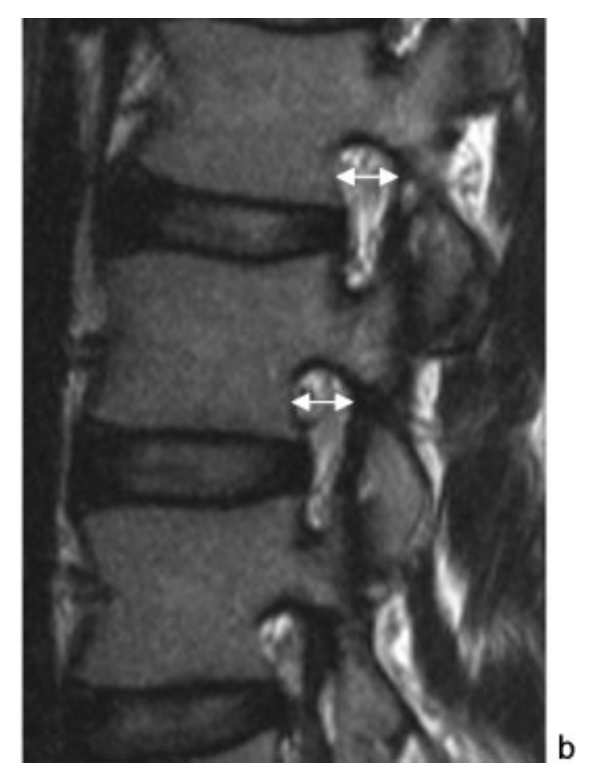

Fig. 12 Measurements of the diameter of the neural foramina. The maximal anteroposterior diameter of the neural foramina is measured on the sagittal images. (a) Schematic drawing; (b) sagittal T2-weighted TSE image.

In the following, the main elements of the classification are summarized (-Fig. 13). For the comprehensive and detailed study of this topic, the entire consensus paper is recommended, which is freely accessible online. By now this classification is accepted among radiologists as well as orthopedists and neurosurgeons and is widely used, rendering it highly recommendable for radiologic diagnosis. The classification is based on the subdivision of the intervertebral disk into four pieces each with a circumference of 90 degrees or $25 \%$ of the whole intervertebral disk circumference. ${ }^{23}$

Bulging is defined as a generalized expansion of the intervertebral disk of $>180$ degrees or $50 \%$ beyond the edges of the vertebral body. Typically the displacement measures $<3 \mathrm{~mm}$. If the expansion prevails to one side, it is described as asymmetric bulging and typically occurs in scolioses due to the unequal distribution of pressure. In contrast a localized displacement of disk material is described as a herniation, if by definition $<180$ degrees or $50 \%$ of the disk circumference is affected. The extent of disk displacement distinguishes a focal herniation $(<90$ degrees or $<25 \%$ ) from a broad-based herniation $(<180$ degrees or $<50 \%)$. ${ }^{23}$

The classification of a herniation as a protrusion or an extrusion depends solely on the size and form of the displaced material (independent of the nature of the displaced tissue). The basis of a protrusion is broader than its diameter toward the spinal canal, noting that this criterion must be observed on sagittal and transverse images. If the diameter of the protrusion toward the spinal canal is greater than its basis, it is considered an extrusion (-Fig. 13). ${ }^{23}$ Furthermore, an extrusion is also present when the displaced disk material overlaps the level of the intervertebral disk on sagittal images. If the displaced disk material shows no contact with the original intervertebral disk, it is called a sequester. Regardless of its proximity to the intervertebral disk, the cranial or caudal displacement of the intervertebral disk tissue on sagittal images is described as migration. If the intervertebral 


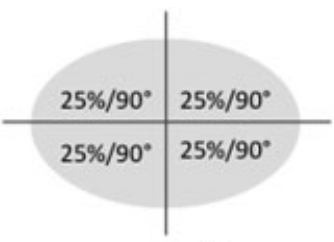

Normal disk

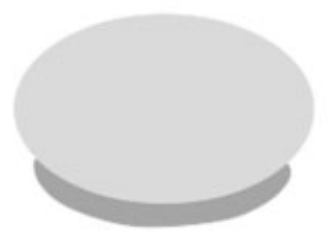

Broad-based herniation

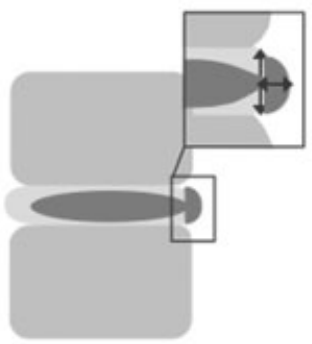

Protrusion

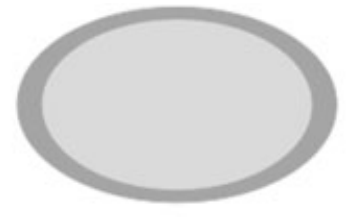

Symmetrical bulging disk

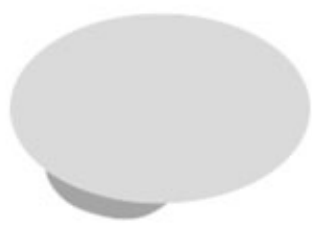

Focal herniation

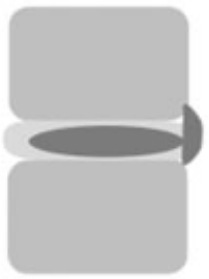

Extrusion (material extends above and/or below disk level

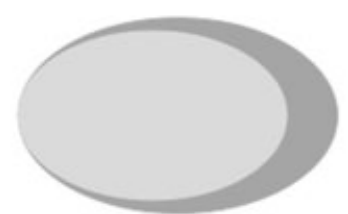

Asymmetrical bulging disk

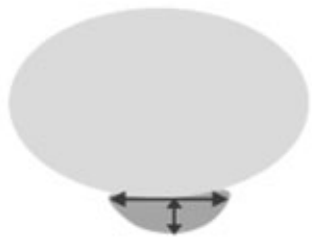

Protrusion

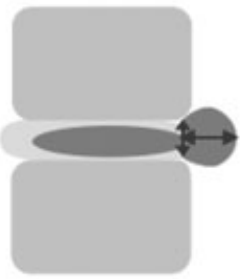

Extrusion

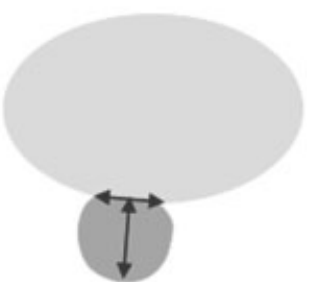

Extrusion

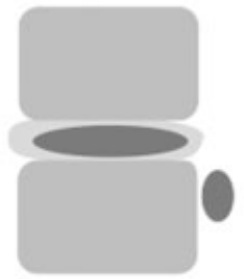

Sequestred fragment

Fig. 13 Nomenclature and classification of lumbar disk pathology according to the North American Spine Society, the American Society of Spine Radiology, and the American Society of Neuroradiology. ${ }^{23}$

tissue is displaced craniocaudally through a defect of the end plate, it is defined as an intervertebral herniation. ${ }^{23}$

For the description of the position of the displaced disk material within the spinal canal, anatomical landmarks are used to define zones on the transverse images and levels on the coronal and sagittal images. ${ }^{23,24}$

The anatomical landmarks, which are used for the classification on the transverse images, are the medial edge of the articular facets as well as the medial and lateral border of the pedicles. The following zones are defined by these landmarks:

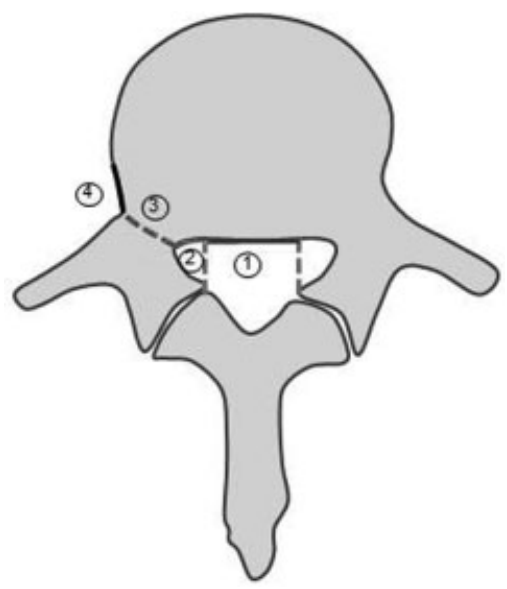

Fig. 14 Anatomical zones for the description of displaced disk material on transverse images as recommended by the classification of the North American Spine Society and other organizations. 1, Central zone; 2 , subarticular zone; 3 , foraminal; 4 , extraforaminal zone. central zone, subarticular zone, foraminal zone, and extraforaminal zone (-Fig. 14) ${ }^{23,24}$ Evaluation of the displaced disk material in the craniocaudal direction involves the following landmarks: the upper and lower borders of the pedicle as well as the upper and lower end plate of the vertebra. In accordance, the following levels are defined on sagittal images: disk level, suprapedicular level, pedicular level, and infrapedicular level (- Fig. 15). ${ }^{23,24}$

\section{Modic Classification}

Degenerative bone marrow changes on MR imaging, caused by osteochondrosis, have been classified by Modic. In the first study published in the year 1988, type 1 and 2 were described, and in a second consecutive study, also published in 1988 , the classification was extended to type $3 .^{25,26}$

Characteristic for type 1 are edematous or reactive inflammatory changes in the vertebral body adjacent to the

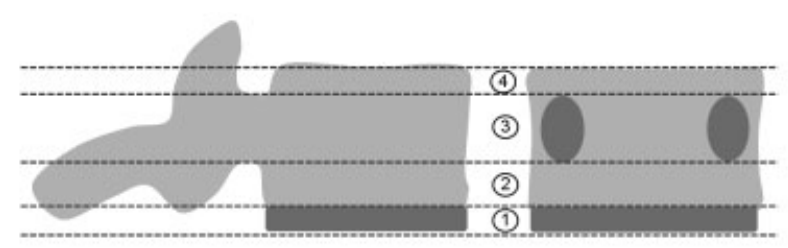

Fig. 15 Anatomical levels for the description of displaced disk material on sagittal and coronal images as recommended by the classification of the North American Spine Society and other organizations. 1, Disk level: 2, infrapendicular level; 3, pedicular level; 4, suprapedicular level. 
intervertebral disk. In accordance a signal intensity decrease is found on the noncontrast T1-weighted images and a signal intensity increase on the T2-weighted images. The signal intensity on the T1-weighted images is characteristically slightly decreased compared with the normal bone marrow, but it does not reach the low signal intensity of the intervertebral disk itself. Type 2 changes are identified as high signal intensities on T2- and T1-weighted images. These changes account for the conversion of hemopoietic bone marrow to fatty bone marrow, most likely caused by the occurrence of ischemia. ${ }^{25,27}$ In type 3, signal intensity decreases on T1- as well as on T2-weighted images, generally attributed to sclerotic changes. ${ }^{26,27}$ Fairly uncommon are findings of different types ( $1 / 2$ and $2 / 3)$ next to each other in one motion segment, which has been interpreted as the transformation from one stage to the next. ${ }^{27,28}$

The clinical significance of the bone marrow changes detected by the MRI has been discussed controversially in the literature. It is commonly accepted that they belong to the framework of the physiologic aging process, characterized by a progression of stages from type 1 to type 3 . Nevertheless, a conversion from the fatty degeneration type 2 to an activated type 1 due to an altered physiologic stress situation is possible. ${ }^{27}$ It is also proven that type 1 changes strongly correlate with lower back pain and segmental instability. Therefore the detection of Modic type 1 in a degeneratively altered segment is an important criterion to evaluate the segment as symptomatic and is considered a strong predictor for a good postoperative outcome after segment stabilization. ${ }^{27}$

\section{References}

1 Weidenbaum M, Shore RM. Radiology in Spinal Deformity-Foundation Knowledge [ebook]. Available at: http://etext.srs.org/book/

2 Waldt S, Eiber M. Spine. In: Waldt S, Woertler K, eds. Measurements and Classification in Musculoskeletal Radiology. Stuttgart and New York: Thieme; 2013:124-148

3 Trobisch PD, Ducoffe AR, Lonner BS, Errico TJ. Choosing fusion levels in adolescent idiopathic scoliosis. J Am Acad Orthop Surg 2013;21(9):519-528

4 Cobb JR. Outline for the study of scoliosis. In: American Academy of Orthopaedic Surgeons, ed. Instructional course lectures. Vol 5. Ann Arbor, MI: Edwards; 1948

5 Ferguson AB. The study and treatment of scoliosis. South Med J 1930;23:116-120

6 Risser JC. The Iliac apophysis; an invaluable sign in the management of scoliosis. Clin Orthop 1958;11(11):111-119

7 Kuntz C. Deformity. In: Chapman JR, Lee MJ, Hermsmeyer JT, et al, eds. Measurements in Spine Care. Stuttgart and New York: Thieme; 2012:319-357

8 Smith JS, Shaffrey CI, Fu KM, et al. Clinical and radiographic evaluation of the adult spinal deformity patient. Neurosurg Clin N Am 2013;24(2):143-156

9 Nash CL Jr, Moe JH. A study of vertebral rotation. J Bone Joint Surg Am 1969;51(2):223-229

10 Lenke LG, Betz RR, Harms J, et al. Adolescent idiopathic scoliosis: a new classification to determine extent of spinal arthrodesis. J Bone Joint Surg Am 2001;83-A(8):1169-1181
11 Bernhardt M, Bridwell KH. Segmental analysis of the sagittal plane alignment of the normal thoracic and lumbar spines and thoracolumbar junction. Spine 1989;14(7):717-721

12 Dihlmann W, Bandick J. Die Gelenksilhouette: das Informationspotenzial der Röntgenstrahlen. Berlin, Germany: Springer; 1995

13 Biggemann M, Frobin W, Brinckmann P. The physiological pattern of lumbar intervertebral disk height. [in German]. Rofo 1997; 167(1):11-15

14 Mamisch N, Brumann M, Hodler J, Held U, Brunner F, Steurer J; Lumbar Spinal Stenosis Outcome Study Working Group Zurich. Radiologic criteria for the diagnosis of spinal stenosis: results of a Delphi survey. Radiology 2012;264(1):174-179

15 Steurer J, Roner S, Gnannt R, Hodler J; LumbSten Research Collaboration. Quantitative radiologic criteria for the diagnosis of lumbar spinal stenosis: a systematic literature review. BMC Musculoskelet Disord 2011;12:175

16 Andreisek G, Hodler J, Steurer J. Uncertainties in the diagnosis of lumbar spinal stenosis. Radiology 2011;261(3):681-684

17 Herzog RJ, Kaiser JA, Saal JA, Saal JS. The importance of posterior epidural fat pad in lumbar central canal stenosis. Spine 1991;16(6, Suppl):S227-S233

18 Hamanishi C, Matukura N, Fujita M, Tomihara M, Tanaka S. Crosssectional area of the stenotic lumbar dural tube measured from the transverse views of magnetic resonance imaging. J Spinal Disord 1994;7(5):388-393

19 Mariconda M, Fava R, Gatto A, Longo C, Milano C. Unilateral laminectomy for bilateral decompression of lumbar spinal stenosis: a prospective comparative study with conservatively treated patients. J Spinal Disord Tech 2002;15(1):39-46

20 Mikhael MA, Ciric I, Tarkington JA, Vick NA. Neuroradiological evaluation of lateral recess syndrome. Radiology 1981;140(1):97-107

21 Strojnik T. Measurement of the lateral recess angle as a possible alternative for evaluation of the lateral recess stenosis on a CT scan. Wien Klin Wochenschr 2001;113(Suppl 3):53-58

22 Beers GJ, Carter AP, Leiter BE, Tilak SP, Shah RR. Interobserver discrepancies in distance measurements from lumbar spine CT scans. AJR Am J Roentgenol 1985;144(2):395-398

23 Fardon DF, Milette PC; Combined Task Forces of the North American Spine Society, American Society of Spine Radiology, and American Society of Neuroradiology. Nomenclature and classification of lumbar disc pathology. Recommendations of the combined task forces of the North American Spine Society, American Society of spine Radiology, and American Society of Neuroradiology. Spine 2001;26(5):E93-E113

24 Wiltse LL, Berger PE, McCulloch JA. A system for reporting the size and location of lesions in the spine. Spine 1997;22(13): 1534-1537

25 Modic MT, Steinberg PM, Ross JS, Masaryk TJ, Carter JR. Degenerative disk disease: assessment of changes in vertebral body marrow with MR imaging. Radiology 1988;166(1 Pt 1):193-199

26 Modic MT, Masaryk TJ, Ross JS, Carter JR. Imaging of degenerative disk disease. Radiology 1988;168(1):177-186

27 Rahme R, Moussa R. The Modic vertebral endplate and marrow changes: pathologic significance and relation to low back pain and segmental instability of the lumbar spine. AJNR Am J Neuroradiol 2008;29(5):838-842

28 Braithwaite I, White J, Saifuddin A, Renton P, Taylor BA. Vertebral end-plate (Modic) changes on lumbar spine MRI: correlation with pain reproduction at lumbar discography. Eur Spine J 1998;7(5): 363-368 